\title{
Plasmonic nanohole electrodes for active color tunable liquid crystal transmissive pixels
}

\author{
Richard Bartholomew ${ }^{1 *}$, Calum Williams ${ }^{1}$, Ammar Khan ${ }^{1}$, Richard Bowman ${ }^{2}$, and Timothy \\ WILKINSON ${ }^{1}$ \\ ${ }^{1}$ Centre of Molecular Materials for Photonics and Electronics, Electrical Engineering Division, Department of Engineering, University of Cambridge, $9 \mathrm{JJ}$ \\ Thomson Avenue, Cambridge CB3 OFA, United Kingdom \\ ${ }^{2}$ Nanophotonics Group, Cavendish Laboratory, University of Cambridge, JJ Thomson Avenue, Cambridge, CB3 OHE, United Kingdom \\ *Corresponding author: rjb92@cam.ac.uk
}

Compiled July 4, 2017

Plasmonic pixels have been shown to offer numerous advantages over pigment-based color filters used in modern commercial liquid crystal (LC) displays. However, wideband dynamic tunability across the visible spectrum remains challenging. We experimentally demonstrate transmissive electrically tunable LCnanohole pixels operating across the visible spectrum with unpolarized input light. An ultrathin Al nanohole electrode is designed to exhibit a polarized spectral response based on surface plasmon resonances. An output analyzer in combination with a nematic LC layer enables pixel color to be electronically controlled through an applied voltage across the device. Where LC reorientation leads to tunable mixing of the relative contributions from the plasmonic color input. The nanostructured Al layer, acting as combined electrode, polarizer and functional color filter, is highly promising for electro-optic display applications. @ 2017 Optical Society of America

OCIS codes: (230.3720) Liquid-crystal devices; (250.5403) Plasmonics; (120.2040) Displays; (350.4238) Nanophotonics and photonic crystals; (310.6628) Subwavelength structures, nanostructures

http://dx.doi.org/10.1364/ao.XX.XXXXXX

Nanoplasmonics offers a pathway for creating a new class of ultrathin display pixel, which exhibit highly tunable optical properties using tailored arrays of sub-wavelength metallic nanostructures [1-3]. Metallic nanohole arrays can be used to excite surface plasmon polaritons (SPPs), leading to wavelength dependent enhanced optical transmission [4]. The optical properties of the nanohole layer are dependant on the geometry of the nanohole and the material of the metallic layer. Through design, SPP excitation can be utilized to create a myriad of plasmonic based pixels operating across the visible spectrum [3, 5-9]. These include multi-color polarization dependent filtering, in which output color is controlled via linear polarizer rotation $[10,11]$, and complex wavefront shaping, based on arrays of spatially variant metallic nanostructures $[12,13]$. Towards the goal of thinner, higher resolution and multi-functional electrooptic display pixels, these plasmonic pixels offer an innovative solution [2]. However, challenges to incorporate wideband tunable optical properties remain [1, 2], and generally, to induce a shift in plasmonic resonance, the manipulation of surrounding materials' refractive index is utilized [3, 14, 15]. Liquid crystals (LCs) are optically anisotropic, and, possessing permanent dipole moments, will re-orientate under an applied electric field, providing an electro-optically tunable medium. Widely used as a fundamental building block for modern displays, LCs are a promising candidate for integration with metallic nanostructures for highly tunable plasmonic pixels $[3,16,17]$.

In this work, we experimentally demonstrate an active color tunable LC-nanohole transmissive pixel device operating in the visible spectrum with unpolarized input light. Through integration of nematic LC with Al nanoholes on glass, an applied voltage across the pixel results in variable color output. The Al nanohole arrays are designed to exhibit plasmonic resonance at a specific wavelength and in effect act to linearly polarise and filter the light. The LCs are used to modulate the polarization state of light as it propagates through the device, followed by a linear polarizer acting to selectively filter the transmission. Using this principal, we show voltage controlled plasmonic color from single pixels. The devices shown are attractive for display applications and may help to reduce the dependency on RGB pigment-based filters, shrink pixel technology and offer additional optical functionality from a single pixel. The devices show advantages over similarly demonstrated electrooptic devices[3,15], such as ease of fabrication, visible spectrum operation and transmissive functionality.

A schematic of the LC-nanohole (LC-NH) pixel is shown in Fig. 1(a). An individual Al nanohole will exhibit localized surface plasmonic resonance (LSPR)[18], spectrally dependent on hole geometry. Whereas, periodic nanohole arrays can provide the additional momentum required to excite propagating SPs, via grating-coupled SPs (GCSPs)[1, 4]. Unlike nano-hole arrays in optically thick metallic layers[19, 20], here GCSPs result in transmission minima[21]. For the former the transmission maxima wavelength is a function of the aspect ratio of the nanohole, the latter, the transmission minima wavelength is proportional 
to array periodicity. As a result, depending on design, the periodic hole arrays will possess both localized and non-localized characteristics. The square nanoholes in this work, under unpolarized light, exhibit two orthogonal modes, shown with finitedifference time-domain (FDTD) simulations [22] in Fig. 1(b): aligned x-polarized $\left(0^{\circ}\right)$ and y-polarized $\left(90^{\circ}\right)$ to the nanohole lattice. The two resonant modes, in an isotropic refractive index $\left(n_{o}=n_{e}\right)$, at any given wavelength, are in phase and of equal intensity. Hence the transmitted light from the nanohole surface has a linearly polarized output (at $45^{\circ}$ ). With LC integration, the surrounding anisotropic index $\left(n_{o} \neq n_{e}\right)$ leads to two distinct modes, as now the surrounding index is dependent due to the orientation of the LC.

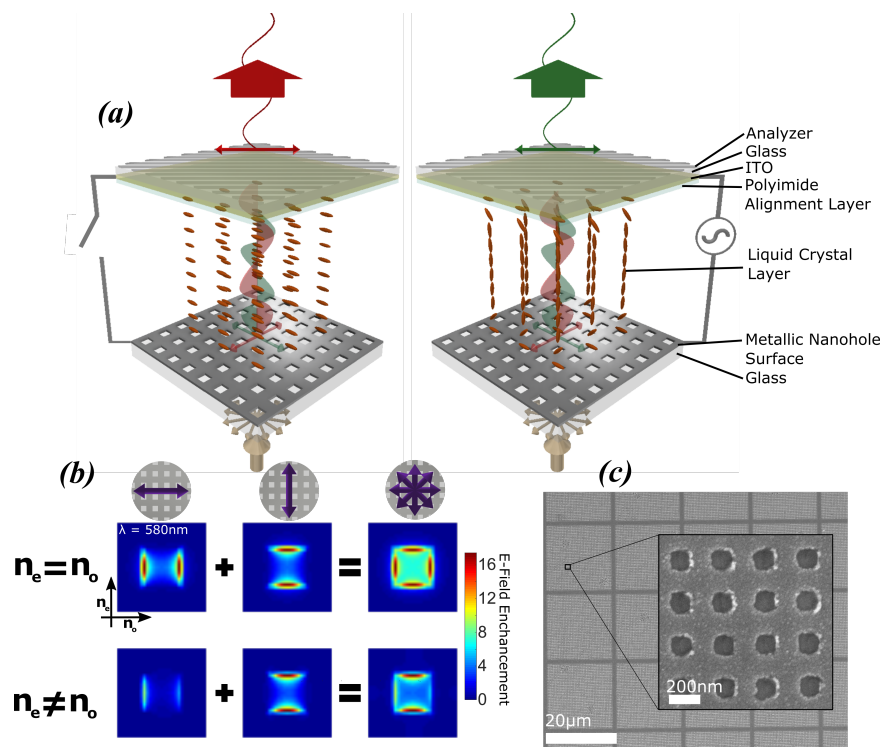

Fig. 1. (a) Schematic of the LC-nanohole device operation: with and without applied voltage. (b) Shows FDTD simulations of the Al nanohole layer $\left(\Lambda_{x / y}=140 \mathrm{~nm}\right.$ and $\left.\Gamma_{x / y}=300 \mathrm{~nm}\right)$ illuminated with un-polarized light. The two orthogonal SP-modes are exhibited shown through the normalized E-field enhancement maps at $\lambda=580 \mathrm{~nm}$. These modes determine the spectra and polarization of light transmitted through the nanohole layer and into the device. When $n_{o}=n_{e}$ both modes are equal and the light is linearly polarized at $45^{\circ}$ to the nanohole lattice. (c) Shows a subset of the fabricated nanohole pixels under SEM.

The devices in this work are constructed from Al (30nm) on glass, patterned using electron-beam lithography to form rectangular nanoholes, shown in Fig. 1(c). Al is an excellent plasmonic material [23], exhibits good chemical stability and is low cost $[8,24]$. Furthermore, it is preferable over other traditional Plasmon materials, such as $\mathrm{Ag}$, due to the preferential planar alignment of LCs to its surface. $80 \mathrm{kV}$ electron beam lithography (EBL) (Nanobeam Ltd. nB1) is used, in conjunction with Ma-N 2405 negative tone photoresist, for the high-resolution patterning of the nanoholes on glass. To overcome the issue of charge accumulation associated with EBL patterning on insulating substrates [25], two charge dissipation layers (2 $\mathrm{nm} \mathrm{Al}$ prior to spin-coating the resist; the second, a $35 \mathrm{~nm}$ Al layer on-top of the resist) are used. Deposition of $\mathrm{Al}(\sim 30 \mathrm{~nm})$ is performed using a thermal evaporator. The Al nanohole array - shown under SEM in Fig. 1(c) - is used as the bottom-electrode of the LC cell (pixel). The top layer of the cell consists of commercial ITO glass with a polyimide layer, mechanically rubbed, giving preferential alignment to the LCs. The cell is constructed using UV-cured glue containing $2 \mu \mathrm{m}$ glass spacer beads resulting in a cell thickness of $2.5-3.3 \mu \mathrm{m}$. The cells were filled with nematic LC BL006 (Merck) in the isotropic phase by heating to $130^{\circ} \mathrm{C}$, phase formation occurring by cooling to room temperature. Optical characterization is with an Olympus BX-51 polarizing optical microscope, with halogen bulb light-source, attached to a spectrometer and DSLR. A $1 \mathrm{kHz}$ sinusoidal AC signal was applied to cell, with $\mathrm{V}_{p-p}$ varying from $0-10 \mathrm{~V}(\Delta V=0.25)$. Full-wave $3 \mathrm{D}$ FDTD modelling is performed using commercial software (Lumerical FDTD Solutions [22]). A single nanohole is modelled with periodic boundary conditions at the unit cell spacing. A complex dispersive material model is used for Al (Palik). An anisotropic refractive index is used to describe a LC-layer with $n_{e}=1.82$ and $n_{o}=1.53$. The voltage dependent index is modelled as a 3D matrix rotation of the LC index, and planar surface anchoring incorporated up to $200 \mathrm{~nm}$ above the nanohole array and below top alignment surface.

The Al nanohole transmission spectra is dependant on the pitch, size and shape of the nanoholes, along with the thickness, density and material of the nanohole layer and surrounding index. The nanohole dimensions utilized were: width, $\Lambda_{x / y}$, ranging from 100-200nm; and pitch, $\Gamma_{x / y}$, ranging from 250450nm (Fig. 3(a)). The pixel is formed encapsulating nematic LCs between the nanohole layer (bottom electrode) and indiumtin-oxide (ITO) covered glass (top electrode). A linearly-rubbed polyimide alignment layer is used on the ITO-glass side only, aligned parallel to the square holes. Nematic LC preferentially align planar to the $\mathrm{Al}$ surface, with bulk LC rotation induced through the top-electrode alignment layer [26]. Bulk LCs reorientate relative to the nanohole surface with applied voltage.

The LC birefringence $\left(\Delta n=n_{\|}-n_{\perp}\right)$ causes the propagating transmitted light from the two nanohole modes to travel through the device at different velocities, altering the phase $\left(\phi_{x / y}\right)$ between the two components. This phase difference $(\gamma$ $\left.=\phi_{x}-\phi_{y}\right)$, at a given wavelength $(\lambda)$, is dependant on the $\Delta n$ and thickness $(t)$ of the LC layer such that $\gamma=2 \pi \Delta n t / \lambda . \Delta n$ between the two modes is dependant on the LC orientation, which is dependent on applied voltage. With no applied field, the LC is planar aligned, whereby $n_{\|}=n_{e}$ and $n_{\perp}=n_{0}$, thus $\Delta n=n_{e}-n_{0}$. Whereas, with an applied voltage (AC signal) sufficiently large enough for homeotropic alignment, $n_{\|}=n_{\perp}=$ $n_{o}$ and $\Delta n=0$. In the intermediary situation, $n_{\|}$is a function of applied voltage, between the two LC indices. This operation is demonstrated through FDTD simulations [22], shown in Fig. 2. The square nanohole array exhibits an optical response dependent on geometry and LC state. As this light propagates through the LC layer, depending on propagation distance, the polarization state oscillates between linearly $(\gamma=0, \pm \pi)$ and circularly $(\gamma= \pm \pi / 2)$ polarized. Thus by varying the amplitude of the applied voltage between the nanohole and ITO layers, the phase difference between propagating orthogonal field components, and the spectra from the LC-NH device, can be controlled.

Moreover, introducing an output analyzer results in an electro-optic device which has an output from a combination of the nanohole array spectra $2 \mathrm{c}$, the optical retardance from the LCs, and output selected to be polarized at $45^{\circ}$ to the nanohole lattice, which can operate in On/Off mode or variable applied voltage color output mode.

Fig. 3 shows the experimental optical transmission results for the LC-NH pixels, for varying nanohole geometries - illustrated in (a). The device is illuminated, with an un-polarized plane wave at normal incidence to the nanohole surface, captured with 


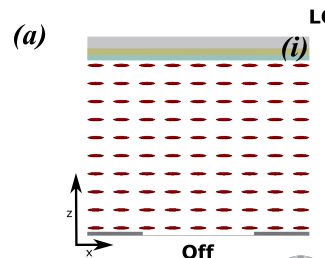

LC Orietation Through Cell
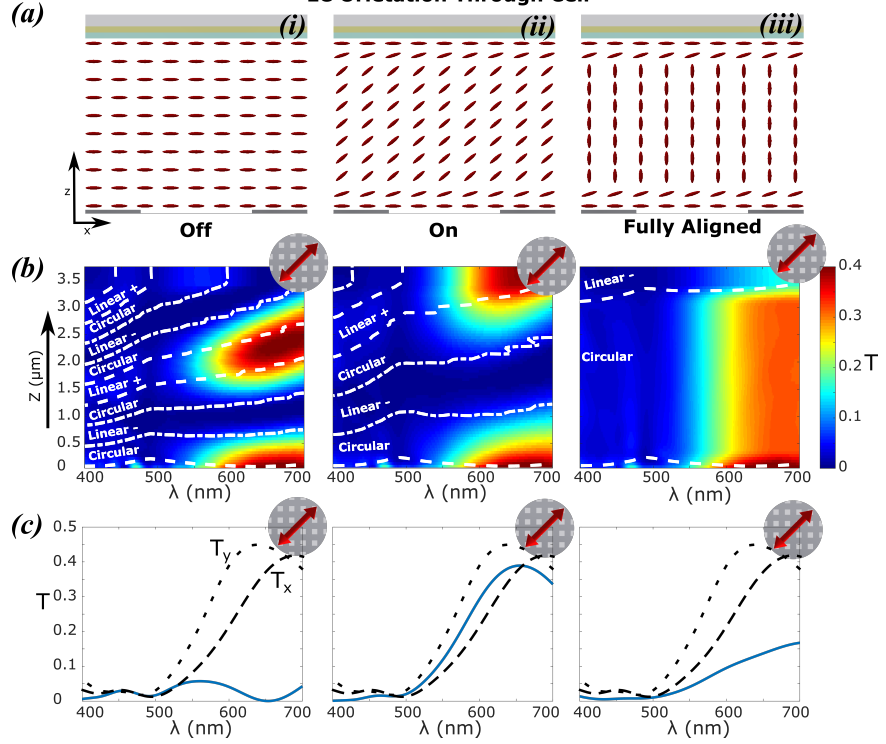

Fig. 2. FDTD simulations of the LC-NH device: $\Lambda_{x / y}=140 \mathrm{~nm}$ and $\Gamma_{x / y}=300 \mathrm{~nm}$. (a) LC orientation (x-z plane) in Off, On and fully aligned states. (b) Transmission spectra, polarized at $45^{\circ}$ (shown with red arrow) to the nanohole lattice, as light propagates through the LC-NH device. (c) Transmission spectra, with output anaylzer at $45^{\circ}$, for the chosen cell thickness of $\sim 3.3$ $\mu \mathrm{m}$. The dashed lines are the unfiltered nanohole response of the two orthogonal SP-modes and solid line post-LC interaction.

both a DSLR (b) and polarizing optical microscope (c-d). An output analyzer is aligned $45^{\circ}$ to the lattice. The device is shown in On/Off $(0 / 4 \mathrm{~V})$ states, and color switching from red to green is visually observed. The total pixel widths are chosen to be large enough $(400 \mu \mathrm{m})$ in order to be easily visible to the naked eye. Fig. 3(c) shows optical microscopy imagery of the LC-NH pixel arrays in the same On/Off states. The pixels contain nanohole arrays with $\Gamma_{x / y}=300 \mathrm{~nm}$ and $\Lambda_{x / y}$ ranging from $110-170 \mathrm{~nm}$. The color variation in the pixels is due to the size variation of the rectangular nanohole geometry.

A single pixel is now selected for investigation into finer control of color - shown in Fig. 3(d). The pixel has fixed nanohole grid geometry: $\Lambda_{x}=155 \mathrm{~nm}, \Lambda_{y}=140 \mathrm{~nm}$ and $\Gamma_{x / y}=300 \mathrm{~nm}$. The applied voltage is varied in incremental steps of $0.5 \mathrm{~V}$. The four rows show images of the selected pixel, with unpolarized illumination, for four output analyzer states: (1) no output analyzer; (2) analyzer orientated at $0^{\circ}$ to the lattice; (3) at $135^{\circ}$; and (4) at $45^{\circ}$. For the unpolarized state, there is no significant change in output color, due to limited re-aligment of LCs around the nanohole surface, resulting in a small shift in plasmonic resonance of the nanohole modes. For an analyzer at $0^{\circ}$, again there is little shift in output color. This analyzer state selects a single orthogonal SP-mode (with reference to Fig 1(b)), and as this mode passes through the LC layer (alignment layer parallel to this analyzer state), regardless of optical retardance, the polarization of the single SP-mode is unaltered. Hence the spectra, filtered by the output analyzer, is unaltered.

For the $45^{\circ} / 135^{\circ}$ state, a combination of the modes can be selected by the output analyzer. The resulting spectra from the two orthogonal analyzer states is such that $T_{\lambda, n o A}=T_{\lambda, A: 45^{\circ}}+$ $T_{\lambda, A: 135^{\circ}}$. The LC layer, through voltage controlled retardance, acts to mix the relative contributions of the two modes and (a)
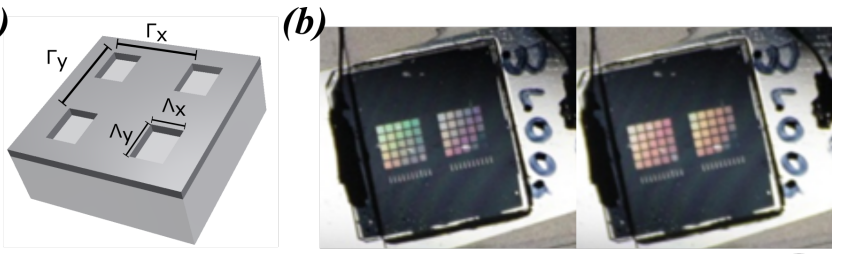

(c)
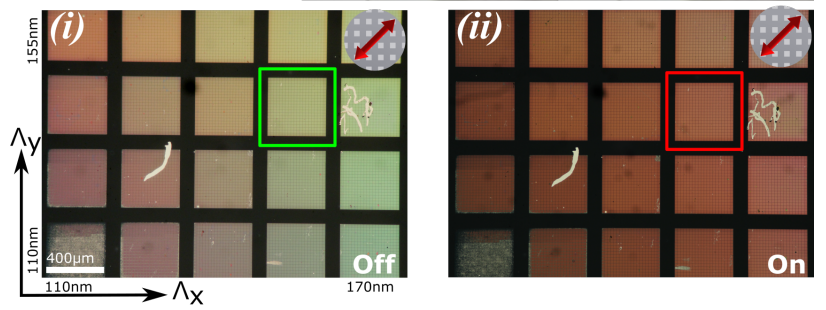

(d)

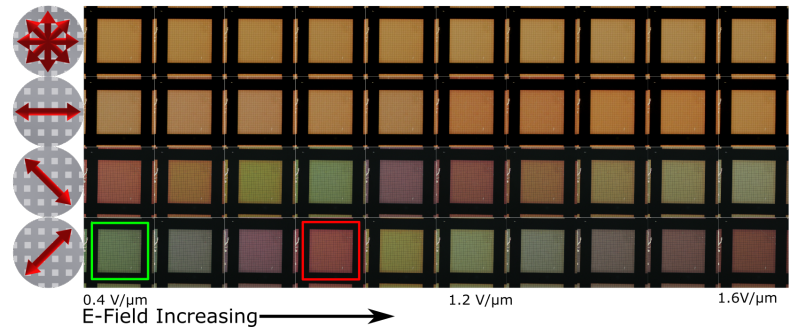

Fig. 3. Experimental optical transmission. (a) Schematic of nanohole surface, indicating width $\left(\Lambda_{x / y}\right)$ and pitch $\left(\Gamma_{x / y}\right) .(b)$ Photos of LC-NH device in Off (left) and On state containing a range of pixels, with $\Gamma_{x / y}=300 \mathrm{~nm}$ and varying $\Lambda_{x / y}$. Under an optical microscope, the same devices are shown in (c), with analyzer at $45^{\circ}$ - the iterated dimensions are indicated on the sub-plot. A single pixel is selected (indicated by colored box) from (c) and the applied voltage is now iterated in smaller increments: results shown in (d). The dimensions are $\Lambda_{x}=155 \mathrm{~nm}$, $\Lambda_{y}=140 \mathrm{~nm}$ and $\Gamma_{x / y}=300 \mathrm{~nm}$. The results are shown with: no output analyzer (top row); output analyzer aligned orthogonal to nanohole lattice; $135^{\circ}$; and $45^{\circ}$ (bottom row).

more specifically acts as a tunable band pass filter. This effect is indicated by the field dependent color shift, shown in Fig. 3(d), and supported through the simulation results in Fig. 2(c).

Fig. 4 shows pixel operation with output analyzer at $135^{\circ}$ in further detail. Two pixel geometries are investigated: $\Gamma_{x / y}=$ $300 \mathrm{~nm}$ and $\Gamma_{x / y}=250 / 350 \mathrm{~nm}$, keeping $\Lambda_{x / y}=140 \mathrm{~nm}$. The experimental transmission spectra for the two LC-NH pixels is shown in Fig. 4(a), whereby two SP modes are observed for orthogonal output states. The pixel in which $\Gamma_{x \neq y}$ results in much greater spectral distinction between orthogonal modes due the variation in orthogonal grating vectors, and hence GCSPs. Fig. 4(b) and Fig. 4(c) show the experimental and simulated transmission spectra respectively for the two LC-NH pixel geometries under varying applied voltage. Fig. 4(d) shows optical microscope images of the respective pixels. Initially the spectra of light is toward the red part of the spectrum. As the electric field increases, the retardance of the LC layer is modulated, and the output transmission peak blue shifts. As the phase difference between the two propagating SP modes cycles between $\gamma=0$ and $\gamma=\pi$ the spectra shifts back and forth from $650 \mathrm{~nm}$ (red) to $550 \mathrm{~nm}$ (green). In the case where $\Gamma_{x / y}=300 \mathrm{~nm}$, both orthogonal SP-modes have their transmission maxima towards the red part of the spectrum, hence red dominated output transmission spectra. At sufficient voltage induced retardance, for which the red part of the spectrum is filtered, the remaining color spectra 

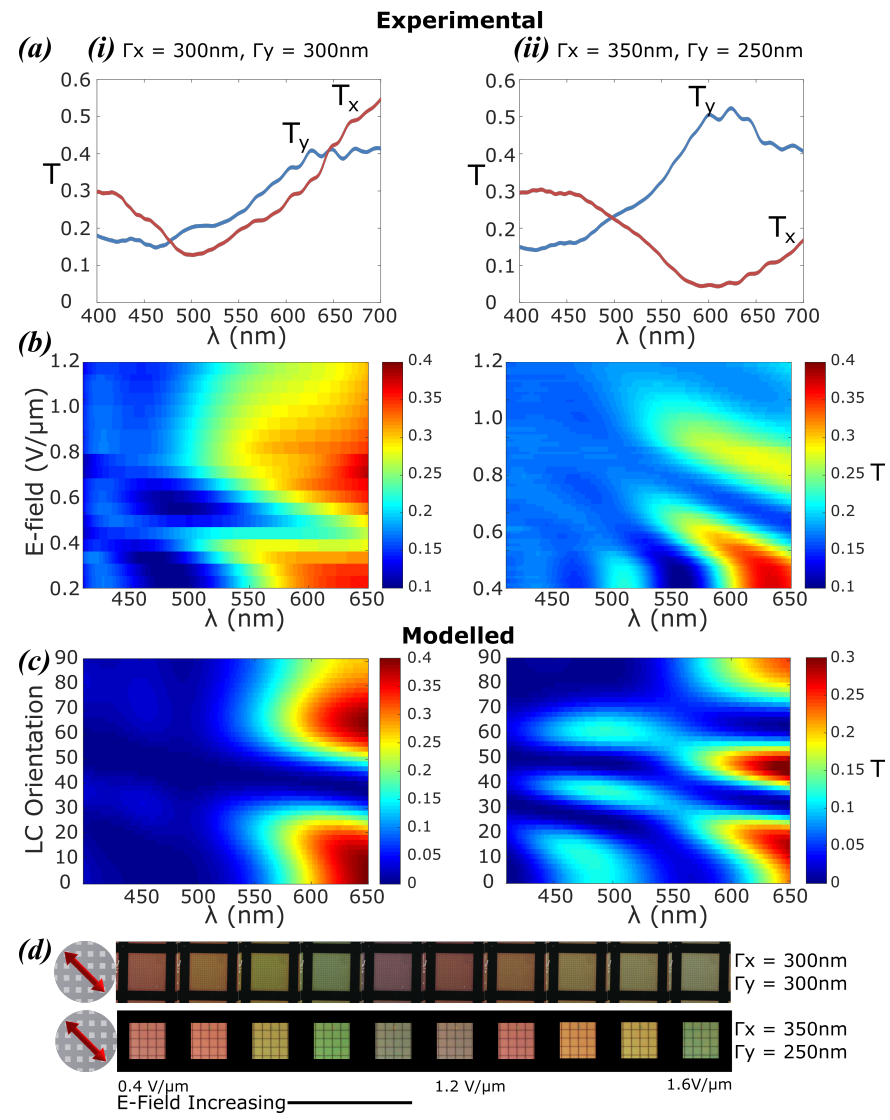

Fig. 4. Experimental and simulated transmission spectra for two LC-NH pixel geometries: (i) $\Gamma_{x=y}=300 \mathrm{~nm}$ and (ii) $\Gamma_{x \neq y}=$ $250 / 350 \mathrm{~nm}$, with constant $\Lambda_{x / y}=140 \mathrm{~nm}$. Pixels are illuminated with unpolarized light and output analyzer at $135^{\circ}$ to the lattice. (a) Experimental transmission spectra from orthogonal nanohole SP modes. (b) Experimental and simulated (c) transmission spectra as a function of voltage. (d) Shows the pixels' color under optical microscope for varying applied voltages.

(in the green part of the spectrum) is broad and weak. Two similar SP-modes are present, with variation in spectral position due in part to the orientation of the LC (anisotropic index) surrounding the nanoholes. Hence the combined output is a broadened red peak. In contrast, for the pixel in which $\Gamma_{x \neq y}$, the more spectrally distinct SP-modes, results in both narrower transmission spectra and a wider color palette over the full tunable range. The two respective pixel outputs are shown in Fig. 4(d) under the same exposure conditions, and in combination with Fig. 4(b-c), demonstrate greater color vividness based on the narrower output peaks. The brightness of the LC-NH pixels may be improved simply by enlarging the size of the nanohole $\left(\Gamma_{x / y}\right)$. The location of the transmission maxima wavelength $\left(\lambda_{p k}\right)$ is dependent on the aspect ratio of the nano-hole, hence the transmission may be increased whilst keeping $\lambda_{p k}$ unchanged. This however comes at a cost, broadening the transmission peak. Any discrepancies between experimental and simulation results is speculated to be due in part to fabrication tolerances (including rounded nanohole geometry, LC surface alignment issues due to resist residue and $\mathrm{Al}$ surface roughness), and simulation methodology inaccuracies (including approximations for LC anchoring and electric field induced LC re-orientation).

In summary, we have experimentally demonstrated an active color tunable LC-NH pixel device operating in the visible spectrum with unpolarized input light. The ultrathin $(<40 \mathrm{~nm}) \mathrm{Al}$ nanohole surface acts to linearly polarize and spectrally filter the input light. The nanohole arrays typically have widths of $140 \mathrm{~nm}$ and periodicity of $300 \mathrm{~nm}$. After propagation through a nematic LC layer, the combined color output is determined through an applied voltage between the nanohole surface and ITO-glass electrodes. The LC retardance actively filters the light passing through pixel in combination with an output analyzer at $45^{\circ}$ to the nanohole lattice. The pixel design combines electrode, polarizer and color filter, reducing the dependency on separate sub-pixel components, such as pigment based-filters, for commercial display devices. [21]

Funding David and Susan Hibbitt Scholarship; Engineering and Physical Sciences Research Council (EPSRC): Integrated Photonic and Electronic Systems (EP/L015455/1)

\section{REFERENCES}

1. Y. Gu, L. Zhang, J. K. W. Yang, S. P. Yeo, and C.-W. Qiu, Nanoscale 7, 6409 (2015).

2. A. Kristensen, J. K. W. Yang, S. I. Bozhevolnyi, S. Link, P. Nordlander, N. J. Halas, and N. A. Mortensen, Nat. Rev. Mater. 2, 16088 (2016).

3. D. Franklin, Y. Chen, A. Vazquez-guardado, S. Modak, J. Boroumand, D. Xu, S.-T. Wu, and D. Chanda, Nat. Commun. 6, 1 (2015).

4. H. F. Ghaemi, T. Thio, T. W. Ebbesen, H. J. Lezec, H. F. Ghaemi, T. Thio, and P. A. Wolff, Nature 391, 667 (1998).

5. K. Kumar, H. Duan, R. S. Hegde, S. C. W. Koh, J. N. Wei, and J. K. W. Yang, Nat. Nanotechnol. 7, 557 (2012).

6. J. Olson, A. Manjavacas, L. Liu, W.-S. Chang, B. Foerster, N. S. King, M. W. Knight, P. Nordlander, N. J. Halas, and S. Link, Proc. Natl. Acad. Sci. U.S.A. 111, 14348 (2014).

7. V. Raj Shrestha, S.-S. Lee, E.-S. Kim, and D.-Y. Choi, Sci. Rep. 5, 12450 (2015).

8. X. M. Goh, Y. Zheng, S. J. Tan, L. Zhang, K. Kumar, C.-W. Qiu, and J. K. W. Yang, Nat. Commun. 5, 5361 (2014).

9. Q. Chen and D. R. S. Cumming, Opt. Express 18, 14056 (2010).

10. L. Duempelmann, A. Luu-Dinh, B. Gallinet, and L. Novotny, ACS Photonics 3, 190 (2016).

11. T. Ellenbogen, K. Seo, and K. B. Crozier, Nano Lett. 12, 1026 (2012).

12. B. Walther, C. Helgert, C. Rockstuhl, F. Setzpfandt, F. Eilenberger, E.-B. Kley, F. Lederer, A. Tünnermann, and T. Pertsch, Adv. Mater. 24, 6300 (2012).

13. C. Williams, Y. Montelongo, J. O. Tenorio-Pearl, A. Cabrero-Vilatela, S. Hofmann, W. I. Milne, and T. D. Wilkinson, Physica Status Solidi Rapid Research Letters 9, 125 (2015).

14. O. Buchnev, J. Y. Ou, M. Kaczmarek, N. I. Zheludev, and V. a. Fedotov, Opt. Express 21, 1633 (2013).

15. O. Buchnev, N. Podoliak, M. Kaczmarek, N. I. Zheludev, and V. A. Fedotov, Adv. Opt. Mater. 3, 674 (2015).

16. I. Abdulhalim, J. Nanophotonics 6, 061001 (2012).

17. J. Olson, A. Manjavacas, T. Basu, D. Huang, A. E. Schlather, B. Zheng, N. J. Halas, P. Nordlander, and S. Link, ACS Nano 10, 1108 (2016).

18. J. Parsons, E. Hendry, C. P. Burrows, B. Auguié, J. R. Sambles, and W. L. Barnes, Phys. Rev. B 79, 073412 (2009).

19. C. Genet and T. W. Ebbesen, Nature 445, 39 (2007).

20. A. Degiron and T. W. Ebbesen, J. Opt. A: Pure Appl. Opt. 7, S90 (2005).

21. Q. G. Du, W. Yue, Z. Wang, W. T. Lau, H. Ren, and E.-P. Li, Opt. Express 24, 4680 (2016).

22. "Lumerical solutions, inc. www.lumerical.com/tcad-products/fdtd/,".

23. P. R. West, S. Ishii, G. V. Naik, N. K. Emani, V. M. Shalaev, and A. Boltasseva, Laser Photon. Rev. 4, 795 (2010).

24. S. J. Tan, L. Zhang, D. Zhu, X. M. Goh, Y. M. Wang, K. Kumar, C.-W. Qiu, and J. K. W. Yang, Nano Lett. 14, 4023 (2014).

25. C. Williams, R. Bartholomew, G. Rughoobur, G. S. D. Gordon, A. J. Flewitt, and T. D. Wilkinson, Nanotechnology 27, 485301 (2016).

26. H. L. Ong, A. J. Hurd, and R. B. Meyer, J. Appl. Phys. 57, 186 (1985). 


\section{FULL REFERENCES}

1. Y. Gu, L. Zhang, J. K. W. Yang, S. P. Yeo, and C.-W. Qiu, "Color generation via subwavelength plasmonic nanostructures." Nanoscale 7, 6409-19 (2015).

2. A. Kristensen, J. K. W. Yang, S. I. Bozhevolnyi, S. Link, P. Nordlander, N. J. Halas, and N. A. Mortensen, "Plasmonic colour generation," Nature Reviews Materials 2, 16088 (2016).

3. D. Franklin, Y. Chen, A. Vazquez-guardado, S. Modak, J. Boroumand, D. Xu, S.-t. Wu, and D. Chanda, "Polarization-independent actively tunable colour generation on imprinted plasmonic surfaces," Nature Communications 6, 1-8 (2015).

4. H. F. Ghaemi, T. Thio, T. W. Ebbesen, H. J. Lezec, H. F. Ghaemi, T. Thio, and P. A. Wolff, "Extraordinary optical transmission through sub-wavelength hole arrays," Nature 391, 667-669 (1998).

5. K. Kumar, H. Duan, R. S. Hegde, S. C. W. Koh, J. N. Wei, and J. K. W. Yang, "Printing colour at the optical diffraction limit," Nature Nanotechnology 7, 557-561 (2012).

6. J. Olson, A. Manjavacas, L. Liu, W.-S. Chang, B. Foerster, N. S. King, M. W. Knight, P. Nordlander, N. J. Halas, and S. Link, "Vivid, full-color aluminum plasmonic pixels," Proceedings of the National Academy of Sciences 111, 14348-14353 (2014).

7. V. Raj Shrestha, S.-S. Lee, E.-S. Kim, and D.-Y. Choi, "Polarizationtuned Dynamic Color Filters Incorporating a Dielectric-loaded Aluminum Nanowire Array." Scientific reports 5, 12450 (2015).

8. X. M. Goh, Y. Zheng, S. J. Tan, L. Zhang, K. Kumar, C.-W. Qiu, and J. K. W. Yang, "Three-dimensional plasmonic stereoscopic prints in full colour," Nature Communications 5, 5361 (2014).

9. Q. Chen and D. R. S. Cumming, "High transmission and low color cross-talk plasmonic color filters using triangular-lattice hole arrays in aluminum films," Optics Express 18, 14056 (2010).

10. L. Duempelmann, A. Luu-Dinh, B. Gallinet, and L. Novotny, "Four-Fold Color Filter Based on Plasmonic Phase Retarder," ACS Photonics 3, 190-196 (2016).

11. T. Ellenbogen, K. Seo, and K. B. Crozier, "Chromatic plasmonic polarizers for active visible color filtering and polarimetry," Nano Letters $\mathbf{1 2}$, 1026-1031 (2012).

12. B. Walther, C. Helgert, C. Rockstuhl, F. Setzpfandt, F. Eilenberger, E.-B. Kley, F. Lederer, A. Tünnermann, and T. Pertsch, "Spatial and spectral light shaping with metamaterials," Advanced Materials 24, 6300-6304 (2012).

13. C. Williams, Y. Montelongo, J. O. Tenorio-Pearl, A. Cabrero-Vilatela, S. Hofmann, W. I. Milne, and T. D. Wilkinson, "Engineered pixels using active plasmonic holograms with liquid crystals," physica status solidi (RRL) - Rapid Research Letters 9, 125-129 (2015).

14. O. Buchnev, J. Y. Ou, M. Kaczmarek, N. I. Zheludev, and V. a. Fedotov, "Electro-optical control in a plasmonic metamaterial hybridised with a liquid-crystal cell." Optics express 21, 1633-8 (2013).

15. O. Buchnev, N. Podoliak, M. Kaczmarek, N. I. Zheludev, and V. A. Fedotov, "Electrically Controlled Nanostructured Metasurface Loaded with Liquid Crystal: Toward Multifunctional Photonic Switch," Advanced Optical Materials pp. n/a-n/a (2015).

16. I. Abdulhalim, "Liquid crystal active nanophotonics and plasmonics: from science to devices," Journal of Nanophotonics 6, 061001 (2012).

17. J. Olson, A. Manjavacas, T. Basu, D. Huang, A. E. Schlather, B. Zheng, N. J. Halas, P. Nordlander, and S. Link, "High chromaticity aluminum plasmonic pixels for active liquid crystal displays," ACS Nano 10, 11081117 (2016).

18. "Lumerical solutions, inc. www.lumerical.com/tcad-products/fdtd/,".

19. P. R. West, S. Ishii, G. V. Naik, N. K. Emani, V. M. Shalaev, and A. Boltasseva, "Searching for better plasmonic materials," Laser and Photonics Reviews 4, 795-808 (2010).

20. H. L. Ong, A. J. Hurd, and R. B. Meyer, "Alignment of nematic liquid crystals by inhomogeneous surfaces," Journal of Applied Physics 57, 186-192 (1985).

21. C. Williams, R. Bartholomew, G. Rughoobur, G. S. D. Gordon, A. J. Flewitt, and T. D. Wilkinson, "Fabrication of nanostructured transmissive optical devices on ITO-glass with UV1116 photoresist using highenergy electron beam lithography," Nanotechnology 27, 485301 (2016). 